\title{
Determination of Antimicrobial Resistance Patterns in Salmonella from Commercial Poultry as Influenced by Microbiological Culture and Antimicrobial Susceptibility Testing Methods
}

\author{
Xi Wang ${ }^{1}$, W. Evan Chaney ${ }^{2}{ }^{(D}$, Hilary O. Pavlidis ${ }^{2}$, James P. McGinnis ${ }^{2}$, J. Allen Byrd ${ }^{2}$, Yuhua Z. Farnell ${ }^{1}{ }^{\mathbb{D}}$, \\ Timothy J. Johnson ${ }^{2}$, Audrey P. McElroy ${ }^{1}$ and Morgan B. Farnell ${ }^{1, * \mathbb{D}}$ \\ 1 Department of Poultry Science, Texas A\&M AgriLife Research, College Station, TX 77843, USA; \\ wangxi@swun.edu.cn (X.W.); yfarnell@tamu.edu (Y.Z.F.); amcelroy@tamu.edu (A.P.M.) \\ 2 Diamond V, Cedar Rapids, IA 52404, USA; echaney@diamondv.com (W.E.C.); \\ hpavlidis@diamondv.com (H.O.P.); jmcginnis@diamondv.com (J.P.M.); \\ Allen.Byrd2@usda.gov (J.A.B.); Tim_Johnson@diamondv.com (T.J.J.) \\ * Correspondence: mfarnell@tamu.edu
}

Citation: Wang, X.; Chaney, W.E.; Pavlidis, H.O.; McGinnis, J.P.; Byrd, J.A.; Farnell, Y.Z.; Johnson, T.J.; McElroy, A.P.; Farnell, M.B. Determination of Antimicrobial Resistance Patterns in Salmonella from Commercial Poultry as Influenced by Microbiological Culture and Antimicrobial Susceptibility Testing Methods. Microorganisms 2021, 9, 1319. https://doi.org/10.3390/ microorganisms 9061319

Academic Editor: Charles M. Dozois

Received: 11 May 2021

Accepted: 12 June 2021

Published: 17 June 2021

Publisher's Note: MDPI stays neutra with regard to jurisdictional claims in published maps and institutional affiliations.

Copyright: (C) 2021 by the authors. Licensee MDPI, Basel, Switzerland. This article is an open access article distributed under the terms and conditions of the Creative Commons Attribution (CC BY) license (https:// creativecommons.org/licenses/by/ $4.0 /)$

\begin{abstract}
Monitoring antimicrobial resistance of foodborne pathogens in poultry is critical for food safety. We aimed to compare antimicrobial resistance phenotypes in Salmonella isolated from poultry samples as influenced by isolation and antimicrobial susceptibility testing methods. Salmonella isolates were cultured from a convenience sample of commercial broiler ceca with and without selective broth enrichment, and resistance phenotypes were determined for 14 antimicrobials using the Sensititre ${ }^{\circledR}$ platform and a qualitative broth breakpoint assay. The broth breakpoint method reported higher resistance to chloramphenicol, sulfisoxazole, and the combination of trimethoprim and sulfamethoxazole, and lower resistance to streptomycin as compared to the Sensititre ${ }^{\circledR}$ assay in trial one. Selective enrichment of samples containing Salmonella in Rappaport-Vassiliadis broth reported lowered detectable resistance to amoxicillin/clavulanic acid, ampicillin, azithromycin, cefoxitin, ceftriaxone, nalidixic acid, and meropenem, and increased resistance to streptomycin and tetracycline than direct-plating samples in trial one. Using matched isolates in trial two, the Sensititre ${ }^{\circledR}$ assay reported higher resistance to chloramphenicol and gentamicin, and lower resistance to nalidixic acid as compared to the broth breakpoint method. These results suggest methodology is a critical consideration in the detection and surveillance of antimicrobial resistance phenotypes in Salmonella isolates from poultry samples and could affect the accuracy of population or industry surveillance insights and intervention strategies.
\end{abstract}

Keywords: antimicrobial resistance; antimicrobial susceptibility testing; poultry; Salmonella

\section{Introduction}

Salmonella is one of the most common foodborne pathogens. The intestinal tract of poultry and other food animals is considered the main foodborne Salmonella reservoir [1,2]. An increasing incidence of antimicrobial resistance (AMR) has been reported in poultry Salmonella isolates where antibiotics are extensively used in production systems [3,4]. Although the link between antimicrobial usage in food animals and clinical treatment failures in human salmonellosis cases remains controversial and inconclusive, the United States Food and Drug Administration (FDA) has fostered antimicrobial stewardship practices to reduce overall resistance since 2018, including ending the use of medically important drugs as growth promoters and requiring a veterinary prescription for medically important drugs [5]. Nevertheless, poultry Salmonella isolates carrying AMR genes have the potential to pass to consumers along the farm-to-fork continuum [6]. Those resistant Salmonella isolates could threaten public health when their resistance phenotypes interfere with drug 
treatments or when they transmit resistance determinants to other pathogens [7]. Monitoring the prevalence and the evolution of foodborne pathogen AMR in poultry and other animals has a critical impact on food safety and public health.

The interagency National Antimicrobial Resistance Monitoring System for enteric bacteria (NARMS) was established in 1996 to track AMR in foodborne pathogens including Salmonella isolated from live production, harvest, and retail products [8]. The NARMS surveillance program tracks Salmonella isolates to determine antibiotic susceptibility as well as resistance genotypes by pulsed-field gel electrophoresis and whole-genome sequencing [9]. A study of 200 Salmonella enterica isolates indicated the correlation of genotypic and phenotypic AMR was $87.61 \%$ and $97.13 \%$ for sensitivity and specificity, respectively [10]. Genotypic-based methods have a promise for rapid detection; however, phenotypic methods have an advantage of accuracy when the resistance is caused by multiple mechanisms [11].

Common antimicrobial susceptibility tests include the agar dilution method, broth dilution methods, gradient diffusion method (on a Mueller-Hinton agar plate), disk diffusion test, and automated instrumentation platforms [12]. The Sensititre ${ }^{\circledR}$ system (TREK Diagnostic Systems, Cleveland, OH, USA) is an automated and standardized method that has been developed and adapted into the NARMS program for surveillance [12]. One isolate, from a positive sample, is selected, prepared, and evaluated for the minimum inhibitory concentration (MIC) level across the entire NARMS panel of antimicrobial compounds per each Sensititre ${ }^{\circledR}$ plate and resistance or susceptibility qualitatively called at the included breakpoint concentration [13]. Usually, a single isolate from a positive sample is submitted and tested through the Sensititre ${ }^{\circledR}$ platform due to expense. Feye and colleagues reported a qualitative broth breakpoint assay to conduct antimicrobial susceptibility testing (AST) of a large number of isolates rapidly and inexpensively [14]. Up to 96 Salmonella isolates, or more depending on plate configurations, can be inoculated into each well of a 96-well plate prefilled with broth and the tested antimicrobial at the Clinical and Laboratory Standards Institute (CLSI)/NARMS recommended resistance breakpoint concentrations [14]. After 18 to $24 \mathrm{~h}$ of incubation, the growth of the inoculated colony could be determined to indicate resistance or susceptibility. While the broth breakpoint method does not produce MIC data, both methods can be compared to estimate resistance prevalence using the current breakpoint concentrations.

We proposed that this broth breakpoint method, facilitating more isolates to be tested, was a more representative means of evaluating phenotypic AMR patterns from a food animal population where foodborne pathogens, such as Salmonella, are expected to be found in much higher prevalence and concentration with greater diversity. Therefore, we conducted two trials to compare phenotypic AMR observations in Salmonella isolated from within a broiler population at harvest as influenced by the culture and AST methodology deployed.

\section{Materials and Methods}

\subsection{Study Design}

Two trials were conducted via a similar workflow (Figure 1). All experimental procedures were reviewed and approved by the Texas A\&M University Institutional Biosafety Committee (IBC number: 2019-073). A convenience sample of ceca was collected from market-aged broilers at harvest provided by a single poultry integrator in the Southeastern United States on 8 December 2018 (trial one) and 27 August 2019 (trial two). Samples were collected in the processing plant and shipped at $2-8{ }^{\circ} \mathrm{C}$ overnight to the laboratory. Upon receipt, samples were prepared and screened by commercial PCR for the presumptive detection of Salmonella enterica (BAX ${ }^{\circledR}$ PCR Assay, Hygiena LLC., Camarillo, CA, USA). Sample retains of presumptive positive samples were then processed through serial dilution and direct-plating onto Salmonella selective agar (brilliant green agar (BGA) with $25 \mu \mathrm{g} \mathrm{mL}{ }^{-1}$ novobiocin in trial one and xylose lysine deoxycholate agar (XLD) in trial two), isolation by secondary selective enrichment in Rappaport-Vassiliadis (RV) broth and 
streak plating to the selective agar plates. Salmonella isolates harvested via direct-plating and enrichment methods were assayed for antimicrobial susceptibility against the NARMS panel of antimicrobials using both the Sensititre ${ }^{\circledR}$ automated system and the broth breakpoint method. The broth assessment was conducted only at the CLSI/NARMS breakpoint concentration of each evaluated antimicrobial compound.
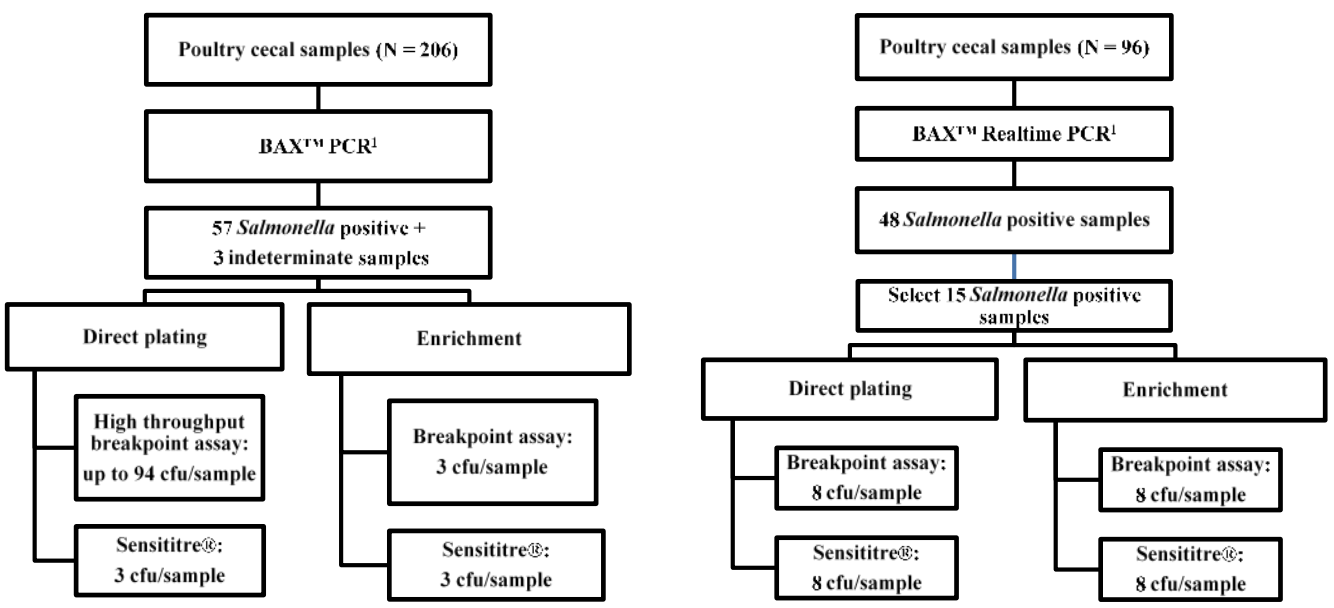

Figure 1. Workflows of trial 1 (left) and trial 2 (right). ${ }^{1}$ Cecum samples in trial one were screened for Salmonella presumptive samples using an endpoint assay (BAX ${ }^{\text {TM }}$ Salmonella 2 PCR Assay, KIT2012, Hygiena LLC), and cecum samples in trial two were screened via a real-time PCR assay (BAX ${ }^{\mathrm{TM}}$ Salmonella Real-time PCR Assay, KIT2006, Hygiena LLC).

In trial one, 57 presumptively positive samples and 3 indeterminate samples were obtained from 206 broiler ceca through PCR screening (Salmonella 2 PCR Assay, KIT2012, Hygiena LLC). These 60 samples were processed with direct plating, and selective enrichment and isolation. From the pool of 60 positive samples, we collected Salmonella isolates from 20 direct plating plates and 38 RV enrichment plates. Up to 94 Salmonella isolates per each of the 20 direct-plating positive samples were tested using the broth breakpoint assay (Figure 1, left). Three isolates from each RV enrichment were also assessed via the breakpoint assay. Another three isolates from the same direct-plating plate and three isolates from the RV enrichment streak plate were processed to perform the Sensititre ${ }^{\circledR}$ method.

For trial two, matched Salmonella isolates were assessed by AST to eliminate the possible effects of isolate sample size and isolate diversity. The interaction and major effects of the culture method and AST method on the phenotypic AMR prevalence was evaluated via a $2 \times 2$ factorial setting of treatments (Figure 1, right). A total of 15 Salmonella positive samples were selected through a PCR screening (Salmonella Real-time PCR Assay, KIT2006, Hygiena LLC), direct-plating, and RV selective enrichment $(n=15)$. A total of 16 isolates ( 8 from direct plating and 8 from selective enrichment) were randomly selected per cecum sample and each individually regrown and evaluated for susceptibility via Sensititre ${ }^{\circledR}$ and the broth breakpoint assays in a paired manner.

\subsection{Sample Preparation}

Cecum samples were collected and packed individually in an enclosed sterile WhirlPak $^{\circledR}$ bag (Nasco, Fort Atkinson, WI, USA) and transported on ice packs at $2-8{ }^{\circ} \mathrm{C}$. Samples were prepared as a slurry by lacerating the entire cecum and massaging the contents in $10 \mathrm{~mL}$ of buffered peptone water (BPW, BD Difco ${ }^{\mathrm{TM}}$, Franklin Lakes, NJ, USA) for $30 \mathrm{~s}$ using a Stomacher ${ }^{\circledR}$ blender (Seward, Bohemia, NY, USA). One mL of the ceca slurry was diluted with nine $\mathrm{mL}$ of BPW to prepare a 1:10 dilution which was then incubated and screened by PCR for Salmonella detection. The remaining slurry was stored at $4 \pm 1{ }^{\circ} \mathrm{C}$ until further analysis. 


\subsection{PCR Screening}

Rapid screening by PCR (BAX ${ }^{\circledR}$ System, Hygiena LLC) was utilized to identify cecum slurries presumptively positive for Salmonella. Briefly, the 1:10 dilution of the slurry was incubated at $37^{\circ} \mathrm{C}$ for $18 \mathrm{~h}$ to pre-enrich bacteria. Bacterial genome fragments were exposed by lysing bacterial cells in $5 \mu \mathrm{L}$ of pre-enriched suspension. The Salmonella target sequence was amplified by the PCR procedure on a Q7 instrument (Hygiena LLC). Trial one utilized an endpoint procedure (BAX ${ }^{\circledR}$ System Salmonella 2, KIT2011, Hygiena LLC) and trial two utilized a real-time detection procedure (BAX ${ }^{\circledR}$ System Real-Time Salmonella Assay, KIT2006, Hygiena LLC). Presumptive presence (positive) or absence (negative) of Salmonella was reported based on the fluorescent signal and software associated with each assay.

\subsection{Salmonella Direct Plating and Enrichment}

Samples presumptively positive by PCR were then cultured for Salmonella in parallel by direct plating of non-enriched sample dilutions and by selective secondary broth enrichment with streak plating for isolation. Direct plating was conducted by a ten-fold serial dilution of the primary cecal slurry using BPW spread plating dilutions onto BGA (trial one) or XLD agar plates (trial two). Selective secondary enrichment and isolation were performed by transferring $100 \mu \mathrm{L}$ of the PCR positive BPW pre-enrichment to $10 \mathrm{~mL}$ of RV broth (Criterion ${ }^{\mathrm{TM}}$, Hardy Diagnostics, Santa Maria, CA, USA) which was incubated at $42 \pm 1{ }^{\circ} \mathrm{C}$ for $24 \mathrm{~h}$. Enriched RV broth was then three-way struck onto selective agar plates for isolation via a $10 \mu \mathrm{L}$ disposable sterile loop (Hach, Loveland, CO, USA). Cecal slurry, dilutions, and enrichment were plated onto BGA (BD Difco ${ }^{\mathrm{TM}}$ ) with $25 \mu \mathrm{g} \mathrm{mL}^{-1}$ novobiocin in trial one and XLD (BD Difco ${ }^{\mathrm{TM}}$ ) agar in trial two. The BGA plated samples were incubated at $37 \pm 1{ }^{\circ} \mathrm{C}$ for $48 \mathrm{~h}$ and the XLD plates for $24 \mathrm{~h}$ followed by room temperature incubation for full-color development. Morphologically typical Salmonella colonies represented as white to red colonies surrounded by red zones on BGA plates and red colonies with black centers on XLD plates were confirmed biochemically (triple sugar iron agar and lysine iron agar, $\mathrm{BD}$ Difco ${ }^{\mathrm{TM}}$ ) and serologically (Salmonella $\mathrm{O}$ antiserum, Poly A-I and Vi, BD Diagnostic, Spark, MD, USA).

\subsection{Broth Breakpoint Assay}

The broth breakpoint assay was modified from a resistance breakpoint assay described by Feye et al. [14]. Briefly, a sterile 96-well plate $\left(\mathrm{VWR}^{\circledR}\right.$ tissue culture plate, Avantor ${ }^{\circledR}$, Wayne, PA, USA) was filled with $200 \mu \mathrm{L}$ of Mueller-Hinton broth (BD Difco ${ }^{\mathrm{TM}}$ ) in each well. A total of 94 colonies along with 2 controls (sterile blank and one Salmonella positive control, wild strain) were individually inoculated into the last two wells by sterilized toothpicks. After overnight incubation at $37 \pm 1^{\circ} \mathrm{C}$, the $\mathrm{OD}_{600}$ absorbance reading (Synergy $\mathrm{H} 1$ plate reader, BioTek Instruments Inc., Winooski, VT, USA) of the plate reached over 0.3 , which corresponded to $3 \times 10^{8}$ cfu of propagated Salmonella $\mathrm{mL}^{-1}$ in each well. The $\mathrm{OD}_{600}$ absorbance reading in the sterile blank was $0.046 \pm 0.001$, which indicated no bacterial growth. Antimicrobials were prepared at the CLSI/NARMS resistance breakpoint concentrations (NARMS panel, Table 1) in Mueller-Hinton broth. The tested antimicrobial solution was aliquoted into $200 \mu \mathrm{L}$ in each well of another sterile 96-well plate for each compound. Propagated Salmonella isolates were inoculated into each antimicrobial plate utilizing a 96 well pin replicator tool (Boekel Scientific ${ }^{\mathrm{TM}}$, Feasterville, PA, USA). The replicator was rinsed, flame sterilized, and cooled before each replicate inoculation. Plates were covered and incubated at $37 \pm 1{ }^{\circ} \mathrm{C}$ for $24 \mathrm{~h}$ and the $\mathrm{OD}_{600}$ reading was recorded. When the absorbance reading was larger than 0.10 , the bacterial growth in the antimicrobial plate was visible and considered resistant at the respective breakpoint. 
Table 1. Resistant breakpoint concentrations of antimicrobial agents used for Salmonella.

\begin{tabular}{cc}
\hline Antimicrobial Agent & Resistant Breakpoint $(\boldsymbol{\mu g} / \mathbf{m L})$ \\
\hline Amoxicillin/Clavulanic Acid ${ }^{\mathbf{1}}$ & $32 / 16$ \\
Ampicillin & 32 \\
Azithromycin & 32 \\
Cefoxitin & 32 \\
Ceftriaxone & 4 \\
Chloramphenicol & 32 \\
Ciprofloxacin & 1 \\
Gentamicin & 16 \\
Meropenem & 4 \\
Nalidixic Acid & 32 \\
Streptomycin & 32 \\
Sulfisoxazole & 512 \\
Tetracycline & 16 \\
Trimethoprim/Sulfamethoxazole ${ }^{2}$ & $4 / 76$ \\
\hline
\end{tabular}

Resistant breakpoint concentrations for Salmonella AMR were cited from the National Antimicrobial Resistant Monitoring System under Disease Control and Prevention (https:/ / www.cdc.gov/narms/antibiotics-tested.html). ${ }^{1}$ the combination of amoxicillin and clavulanic acid was prepared at a 1:2 ratio with $32 \mu \mathrm{g} / \mathrm{mL}$ of amoxicillin and $16 \mu \mathrm{g} / \mathrm{mL}$ of clavulanic acid in the final testing well. ${ }^{2}$ the combination of trimethoprim and sulfamethoxazole was prepar6ed at 1:19 ratio with $4 \mu \mathrm{g} / \mathrm{mL}$ of trimethoprim and $76 \mu \mathrm{g} / \mathrm{mL}$ of sulfamethoxazole in the final testing well.

\subsection{Automated AST System}

The Sensititre ${ }^{\circledR}$ platform was utilized following the manufacturer's instructions. Antimicrobial susceptibility testing of Salmonella was determined using the CMV4AGNF susceptibility plate (Thermo Fisher Scientific, Waltham, MA, USA). Briefly, an individual isolate was randomly chosen from a direct-plating or enrichment, struck onto a blood agar plate (Trypticase ${ }^{\mathrm{TM}}$ soy agar with $5 \%$ sheep blood, BD BBL ${ }^{\mathrm{TM}}$, Sparks, MD), and incubated at $35 \pm 1{ }^{\circ} \mathrm{C}$ for $24 \mathrm{~h}$. To achieve the optimal concentration, one or two pure colonies were suspended into five $\mathrm{mL}$ of deionized water and adjusted to a $0.5 \mathrm{McF}$ arland standard equivalent using a Sensititre ${ }^{\circledR}$ nephelometer (Thermo Scientific ${ }^{\mathrm{TM}}$ ), and $10 \mu \mathrm{L}$ of the suspension was transferred to $11 \mathrm{~mL}$ of Mueller-Hinton broth (Thermo Scientific ${ }^{\mathrm{TM}}$ ). Fifty $\mu \mathrm{L}$ of the broth was inoculated into each well of the Sensititre ${ }^{\circledR}$ plate by an automated inoculation delivery system (Sensititre $\mathrm{AIM}^{\mathrm{TM}}$, Thermo Scientific ${ }^{\mathrm{TM}}$ ) and incubated at $37 \pm 1{ }^{\circ} \mathrm{C}$ for $24 \mathrm{~h}$. Sensititre ${ }^{\circledR}$ plates were read automatically (OptiRead ${ }^{\mathrm{TM}}$ Automated Fluorometric Plate Reading System, Thermo Scientific ${ }^{\mathrm{TM}}$ ) and confirmed manually (Vizion ${ }^{\mathrm{TM}}$ Digital MIC Viewing System, Thermo Scientific ${ }^{\mathrm{TM}}$ ). The growth of the colony in the tested antimicrobial solution at the breakpoint resistance concentration was determined for resistance.

\subsection{Data Analysis}

In trial one, AMR prevalence (\% resistant isolates) outcomes observed by the two AST methods were compared by Chi-square analysis using the SAS FREQ procedure in trial one [15]. To reduce the possible effects of sampling size on AMR, AMR prevalence outcomes of the equal number of Salmonella isolates were randomly selected from the broth breakpoint assay and then compared to the Sensititre ${ }^{\mathrm{TM}}$ assay. Randomization was conducted by a random number producer in Excel (Microsoft, Redmond, WA, USA). In trial one, 14 cecum slurry samples were positive for Salmonella colonies by direct plating and enrichment. Prevalence of resistance generated from the Sensititre ${ }^{\circledR}$ method was compared for differences between direct plating and enrichment using a nonparametric method, the Wilcoxon signed-rank test in SAS NPAR1WAY procedure. In trial two, the major effects of susceptibility testing methodology and culturing methodology and their interactions on poultry Salmonella AMR were evaluated. A two-way ANOVA analysis was conducted by the SAS GLM procedure. All significance levels were set at $\alpha=0.05$. 


\section{Results}

In trial one, 206 broiler cecum samples were screened by PCR assay and presumptive samples were cultured in parallel by direct plating and selective enrichment methods. Fifty-seven samples were PCR positive and three were indeterminate. Two of the three indeterminate samples were confirmed to be positive through cultural isolation, which indicated Salmonella prevalence in the convenience sample broiler population was approximately $28.6 \%$ (59/206, either PCR or culture positive).

From the above positive samples, a total of 1748 Salmonella isolates collected from 20 direct plating samples were processed by the broth breakpoint assay and 55 isolates from 20 direct plating samples were analyzed by the Sensititre ${ }^{\circledR}$ method. Five enrichment isolates were not recoverable when transferred to the 96 well plates containing $\mathrm{MH}$ broth. Effects of antimicrobial susceptibility methods on Salmonella AMR patterns are reported in Table 2. As compared to the Sensititre ${ }^{\circledR}$ method, the broth breakpoint method reported higher resistance to chloramphenicol (60.3\% vs. $25.5 \%)$, ciprofloxacin $(16.7 \%$ vs. 5.5\%), sulfisoxazole (61.9\% vs. 9.1\%), and the combination of trimethoprim and sulfamethoxazole $(65.9 \%$ vs. $12.7 \%)$, and lower resistance to azithromycin (15.5\% vs. $32.7 \%)$, meropenem $(16.5 \%$ vs. $43.6 \%)$, and streptomycin $(13.4 \%$ vs. $32.7 \%)$ in poultry Salmonella (all $p<0.05)$.

Table 2. Effects of antimicrobial susceptibility testing methodology on resistance ratios of poultry Salmonella collected via direct plating in trial one.

\begin{tabular}{|c|c|c|c|c|}
\hline \multirow{2}{*}{ Antibiotics } & Broth Breakpoint & Sensititre $^{\circledR}$ & \multirow{2}{*}{$X^{2}$} & \multirow{2}{*}{$p$ Value } \\
\hline & $n=1748$ & $n=55$ & & \\
\hline Amoxicillin/Clavulanic acid & $1140(63.9 \%)$ & $32(58.2 \%)$ & 0.755 & 0.396 \\
\hline Ampicillin & $1173(65.8 \%)$ & $39(70.9 \%)$ & 0.632 & 0.473 \\
\hline Azithromycin & $277(15.5 \%)$ & $18(32.7 \%)$ & 11.72 & 0.002 \\
\hline Cefoxitin & $1344(75.3 \%)$ & $37(67.3 \%)$ & 1.86 & 0.204 \\
\hline Ceftriaxone & $1129(63.3 \%)$ & $31(56.4 \%)$ & 1.09 & 0.322 \\
\hline Chloramphenicol & $1075(60.3 \%)$ & $14(25.5 \%)$ & 26.76 & $<0.001$ \\
\hline Ciprofloxacin & $298(16.7 \%)$ & $3(5.5 \%)$ & 4.93 & 0.025 \\
\hline Gentamicin & $113(6.33 \%)$ & $3(5.5 \%)$ & 0.070 & 0.990 \\
\hline Nalidixic Acid & $477(26.7 \%)$ & $12(21.8 \%)$ & 0.662 & 0.535 \\
\hline Meropenem & $295(16.5 \%)$ & $24(43.6 \%)$ & 27.33 & $<0.001$ \\
\hline Streptomycin & $239(13.4 \%)$ & $18(32.7 \%)$ & 16.58 & $<0.001$ \\
\hline Sulfisoxazole & $1104(61.9 \%)$ & $5(9.1 \%)$ & 62.12 & $<0.001$ \\
\hline Tetracycline & $393(22.0 \%)$ & $13(23.6 \%)$ & 0.080 & 0.743 \\
\hline Trimethoprim/Sulfamethoxazole & $1176(65.9 \%)$ & $7(12.7 \%)$ & 65.79 & $<0.001$ \\
\hline
\end{tabular}

Salmonella isolates were collected from directly plating 20 positive poultry ceca dilutions onto BGA agar plates in trial one. Up to 94 Salmonella isolates per cecum sample were tested via the broth breakpoint assay and 1748 isolates were recovered. Three Salmonella isolates per cecum sample were randomly selected and tested using the Sensititre ${ }^{\circledR}$ system.

To reduce the effects of sampling size of isolates on Salmonella AMR between AST methods, an equal number of isolates ( $55 \mathrm{cfu}$ ) were randomly selected from the broth breakpoint method and their AMR profiles were compared to those reported by the Sensititre ${ }^{\circledR}$ system (Table 3). Similar results were observed here between the broth breakpoint method and the Sensititre ${ }^{\circledR}$ system, as compared to the comparisons above. As compared to the Sensititre ${ }^{\circledR}$ method, the broth breakpoint method reported higher resistance to chloramphenicol (60.0\% vs. $25.5 \%)$, ciprofloxacin (27.3\% vs. $5.5 \%)$, sulfisoxazole $(72.7 \%$ vs. $9.1 \%)$, and the combination of trimethoprim and sulfamethoxazole (61.8\% vs. $12.7 \%)$, and lower resistance to azithromycin (14.5\% vs. $32.7 \%)$, meropenem (12.7\% vs. $43.6 \%)$, and streptomycin $(12.7 \%$ vs. $32.7 \%$ ) in poultry Salmonella (all $p<0.05)$. Randomized selections and comparisons were conducted five times and similar results were generated (data not shown). 
Table 3. Effects of antimicrobial susceptibility testing methodology on resistance ratios of poultry Salmonella collected via direct plating in trial one (equal sample size).

\begin{tabular}{|c|c|c|c|c|}
\hline \multirow{2}{*}{ Antibiotics } & Broth Breakpoint & Sensititre ${ }^{\circledR}$ & \multirow{2}{*}{$X^{2}$} & \multirow{2}{*}{$p$ Value } \\
\hline & $n=55$ & $n=55$ & & \\
\hline Amoxicillin/Clavulanic acid & $33(60.0 \%)$ & $32(58.2 \%)$ & 0.038 & 0.999 \\
\hline Ampicillin & $32(58.2 \%)$ & $39(70.9 \%)$ & 1.96 & 0.232 \\
\hline Azithromycin & $8(14.5 \%)$ & $18(32.7 \%)$ & 5.04 & 0.043 \\
\hline Cefoxitin & $41(74.5 \%)$ & $37(67.3 \%)$ & 0.705 & 0.529 \\
\hline Ceftriaxone & $33(60.0 \%)$ & $31(56.4 \%)$ & 0.149 & 0.847 \\
\hline Chloramphenicol & $33(60.0 \%)$ & $14(25.5 \%)$ & 13.41 & $<0.001$ \\
\hline Ciprofloxacin & $15(27.3 \%)$ & $3(5.5 \%)$ & 9.56 & 0.004 \\
\hline Gentamicin & $4(7.3 \%)$ & $3(5.5 \%)$ & 0.153 & 0.999 \\
\hline Nalidixic Acid & $14(25.5 \%)$ & $12(21.8 \%)$ & 0.202 & 0.823 \\
\hline Meropenem & $7(12.7 \%)$ & $24(43.6 \%)$ & 12.98 & $<0.001$ \\
\hline Streptomycin & $7(12.7 \%)$ & $18(32.7 \%)$ & 6.26 & 0.022 \\
\hline Sulfisoxazole & $40(72.7 \%)$ & $5(9.1 \%)$ & 46.06 & $<0.001$ \\
\hline Tetracycline & $16(29.1 \%)$ & $13(23.6 \%)$ & 0.422 & 0.666 \\
\hline Trimethoprim/Sulfamethoxazole & $34(61.8 \%)$ & $7(12.7 \%)$ & 28.35 & $<0.001$ \\
\hline
\end{tabular}

Salmonella isolates were collected from directly plating 20 positive poultry ceca dilutions onto BGA agar plates in trial one. Up to 94 Salmonella isolates per cecum sample were tested via the broth breakpoint assay and 55 isolates were randomly selected for comparison. Three Salmonella isolates per cecum sample were randomly selected and tested using the Sensititre ${ }^{\circledR}$ system. Randomization was conducted five times and similar comparison results were generated.

A total of 222 isolates were cultured from 38 samples via RV selective secondary enrichment and assayed for susceptibility in the broth breakpoint assay (108 isolates, Table 4) and the Sensititre ${ }^{\circledR}$ method (114 isolates). The broth breakpoint assay reported a higher prevalence of resistance to the combination of amoxicillin and clavulanic acid ( $32.1 \%$ vs. $5.3 \%)$, ampicillin ( $56.6 \%$ vs. $5.3 \%)$, cefoxitin ( $59.4 \%$ vs. $5.3 \%)$, chloramphenicol ( $67 \%$ vs. $5.3 \%)$, meropenem $(74.5 \%$ vs. $5.3 \%)$, sulfisoxazole ( $98.1 \%$ vs. $5.3 \%)$, and the combination of trimethoprim and sulfamethoxazole (31.1\% vs. $5.3 \%)$ and lower prevalence of resistance to streptomycin $(75.5 \%$ vs. $97.4 \%$, all $p<0.050)$ when compared to the Sensititre ${ }^{\circledR}$ method, respectively.

Table 4. Effects of antimicrobial susceptibility testing methodology on resistance ratios of poultry Salmonella isolated via selective enrichment in trial one.

\begin{tabular}{ccccc}
\hline & Broth Breakpoint & Sensititre & & \\
\cline { 2 - 3 } Antibiotics & $\boldsymbol{n}=\mathbf{1 0 8}$ & $\boldsymbol{n}=\mathbf{1 1 4}$ & $\boldsymbol{X}^{\mathbf{2}}$ & $\boldsymbol{p}$ Value \\
\cline { 2 - 3 } Amoxicillin/Clavulanic acid & $34(32.1 \%)$ & $6(5.3 \%)$ & 26.54 & $<0.001$ \\
Ampicillin & $60(56.6 \%)$ & $6(5.3 \%)$ & 68.94 & $<0.001$ \\
Azithromycin & $13(12.3 \%)$ & $6(5.3 \%)$ & 3.41 & 0.091 \\
Cefoxitin & $63(59.4 \%)$ & $6(5.3 \%)$ & 74.88 & $<0.001$ \\
Ceftriaxone & $5(4.7 \%)$ & $9(7.9 \%)$ & 0.93 & 0.413 \\
Chloramphenicol & $71(67.0 \%)$ & $6(5.3 \%)$ & 91.97 & $<0.001$ \\
Ciprofloxacin & $4(3.8 \%)$ & $0(0 \%)$ & $\mathrm{NA}$ & $\mathrm{NA}$ \\
Gentamicin & $0(0 \%)$ & $0(0 \%)$ & $\mathrm{NA}$ & $\mathrm{NA}$ \\
Nalidixic Acid & $6(5.7 \%)$ & $0(0 \%)$ & $\mathrm{NA}$ & $\mathrm{NA}$ \\
Meropenem & $79(74.5 \%)$ & $6(5.3 \%)$ & 111.15 & $<0.001$ \\
Streptomycin & $80(75.5 \%)$ & $111(97.4 \%)$ & 23.01 & $<0.001$ \\
Sulfisoxazole & $104(98.1 \%)$ & $6(5.3 \%)$ & 189.41 & $<0.001$ \\
Tetracycline & $106(100 \%)$ & $109(95.6 \%)$ & $\mathrm{NA}$ & NA \\
Trimethoprim/Sulfamethoxazole & $33(31.1 \%)$ & $6(5.3 \%)$ & 25.20 & $<0.001$
\end{tabular}

NA: not available. When $50 \%$ of the cells have expected counts less than five, Chi-square is not a valid test. Salmonella isolates were collected from 38 poultry ceca enrichments (RV) in trial one. Three Salmonella isolates per cecum sample were tested via the broth breakpoint assay and another three isolates via the Sensititre ${ }^{\circledR}$ method. Six colonies, transferred from the enrichment plates, did not grow when passaged to the Mueller-Hinton containing 96-well plates and only 108 colonies were tested for AMR via broth breakpoint assay. 
A nonparametric analysis was conducted to analyze the effects of culturing method on the Salmonella AMR pattern generated from the Sensititre ${ }^{\circledR}$ platform $(n=14$, Table 5$)$. Salmonella isolates collected from direct plating demonstrated higher resistance to the combination amoxicillin and clavulanic acid, ampicillin, azithromycin, cefoxitin, ceftriaxone, nalidixic acid, and meropenem, and lower resistance to streptomycin and tetracycline.

Table 5. Effects of culturing method on poultry Salmonella antimicrobial resistance in trial one.

\begin{tabular}{cccc}
\hline & \multicolumn{2}{c}{ Wilcoxon Score } & \\
\cline { 2 - 3 } Antibiotics & Direct Plating & Enrichment & $p$ Value \\
\hline Amoxicillin/Clavulanic acid & 18.93 & 10.07 & 0.001 \\
Ampicillin & 18.96 & 10.04 & 0.001 \\
Azithromycin & 17.36 & 11.64 & 0.023 \\
Cefoxitin & 18.96 & 10.04 & 0.001 \\
Ceftriaxone & 18.89 & 10.11 & 0.001 \\
Chloramphenicol & 16.39 & 12.61 & 0.096 \\
Ciprofloxacin & 15.00 & 14.00 & 0.353 \\
Gentamicin & 15.50 & 13.50 & 0.165 \\
Nalidixic Acid & 17.00 & 12.00 & 0.017 \\
Meropenem & 17.86 & 11.14 & 0.010 \\
Streptomycin & 9.29 & 19.71 & $<.001$ \\
Sulfisoxazole & 14.96 & 14.04 & 0.608 \\
Tetracycline & 8.61 & 20.39 & $<.001$ \\
Trimethoprim/Sulfamethoxazole & 15.89 & 13.11 & 0.191 \\
\hline
\end{tabular}

Resistance colony counts through the Sensititre ${ }^{\circledR}$ assay from direct plating and those counts from RV enrichment were paired through the Wilcoxon ranking test ( $n=14$ birds). Mean Wilcoxon scores and $p$-values were presented.

In trial two, 96 broiler ceca were screened and generated 48 presumptive positive samples. The 48 presumptive samples were processed for direct plating and RV enrichment. Each sample grew on XLD agar plates with morphologies typical of Salmonella, indicating a 50\% (48/96) incidence in our convenience samples.

Fifteen samples were randomly selected and assayed for AMR through both AST methods in trial two. To eliminate the effects of Salmonella isolate diversity on AST outcomes, each randomly selected Salmonella isolate was struck onto a new agar plate and the same colonies were introduced into the breakpoint assay and Sensititre ${ }^{\circledR}$ assay in parallel. Major effects and interactions of the susceptibility test and culturing methodology on Salmonella AMR are reported in Table 6. After accounting for isolate diversity between AST methods, neither the interaction nor the culturing method affected Salmonella AMR observations. The broth breakpoint assay reported lower resistance to chloramphenicol $(0 \%$ vs. $2.08 \%)$, gentamicin ( $0 \%$ vs. $3.75 \%)$, and higher resistance to nalidixic acid (5.0\% vs. $0.83 \%$ ) as compared to the Sensititre ${ }^{\circledR}$ method (all $p<0.05$ ).

Table 6. Effects of antimicrobial resistance methodology and bacteria culturing method on Salmonella antimicrobial resistance patterns in trial two.

\begin{tabular}{|c|c|c|c|c|c|c|c|c|c|}
\hline & & & & & & & & $p$ Value & \\
\hline Methodology & Sensititre $^{\circledR}$ & $\begin{array}{c}\text { Broth } \\
\text { Breakpoint }\end{array}$ & SEM & $\begin{array}{l}\text { Direct } \\
\text { Plating }\end{array}$ & Enrichment & SEM & Method & Culture & $\begin{array}{c}\text { Method } \times \\
\text { Culture }\end{array}$ \\
\hline $\begin{array}{c}\text { Amoxicillin/ } \\
\text { Clavulanic acid }\end{array}$ & $12.1 \%$ & $10.0 \%$ & $3.65 \%$ & $10.8 \%$ & $11.3 \%$ & $3.65 \%$ & 0.688 & 0.936 & 0.574 \\
\hline Ampicillin & $7.9 \%$ & $2.9 \%$ & $2.16 \%$ & $5.8 \%$ & $5.0 \%$ & $2.16 \%$ & 0.107 & 0.786 & 0.587 \\
\hline Azithromycin & $35.8 \%$ & $37.9 \%$ & $7.79 \%$ & $43.3 \%$ & $30.4 \%$ & $7.79 \%$ & 0.851 & 0.246 & 0.910 \\
\hline Ceftriaxone & $5.4 \%$ & $0.8 \%$ & $1.96 \%$ & $2.9 \%$ & $3.3 \%$ & $1.96 \%$ & 0.103 & 0.881 & 0.881 \\
\hline Cefoxitin & $0.4 \%$ & $0.0 \%$ & $0.30 \%$ & $0.0 \%$ & $0.4 \%$ & $0.30 \%$ & 0.322 & 0.322 & 0.322 \\
\hline Chloramphenicol & $2.08 \%{ }^{a}$ & $0.00 \% \mathrm{~b}$ & $0.62 \%$ & $0.8 \%$ & $1.3 \%$ & $0.62 \%$ & 0.021 & 0.637 & 0.637 \\
\hline Gentamicin & $3.75 \%$ a & $0.00 \% \mathrm{~b}$ & $1.03 \%$ & $2.9 \%$ & $0.8 \%$ & $1.03 \%$ & 0.013 & 0.159 & 0.159 \\
\hline Meropenem & $2.5 \%$ & $4.2 \%$ & $1.73 \%$ & $1.7 \%$ & $5.0 \%$ & $1.73 \%$ & 0.498 & 0.178 & 0.498 \\
\hline Nalidixic acid & $0.83 \% \mathrm{~b}$ & $5.00 \%{ }^{a}$ & $1.31 \%$ & $3.3 \%$ & $2.5 \%$ & $1.31 \%$ & 0.028 & 0.654 & 0.182 \\
\hline
\end{tabular}


Table 6. Cont.

\begin{tabular}{|c|c|c|c|c|c|c|c|c|c|}
\hline & & & & & & \multicolumn{4}{|c|}{$p$ Value } \\
\hline Methodology & Sensititre $^{\circledR}$ & $\begin{array}{c}\text { Broth } \\
\text { Breakpoint }\end{array}$ & SEM & $\begin{array}{l}\text { Direct } \\
\text { Plating }\end{array}$ & Enrichment & SEM & Method & Culture & $\begin{array}{c}\text { Method } \times \\
\text { Culture }\end{array}$ \\
\hline Streptomycin & $72.1 \%$ & $75.0 \%$ & $5.43 \%$ & $70.4 \%$ & $76.7 \%$ & $5.43 \%$ & 0.705 & 0.419 & 0.705 \\
\hline $\begin{array}{c}\text { Trimethoprim/ } \\
\text { Sulphamethoxazole }\end{array}$ & $4.6 \%$ & $4.6 \%$ & $3.35 \%$ & $8.3 \%$ & $0.8 \%$ & $3.35 \%$ & 0.999 & 0.119 & 0.990 \\
\hline
\end{tabular}

a,b different subscripts in a row stand for a significant difference at $\alpha=0.05$. Means from interactions are not listed due to a lack of significant difference. A total of 15 Salmonella positive broiler cecal samples were selected in trial two. For each cecum sample, eight isolates from direct plating and another eight from enrichment were tested by the Sensititre ${ }^{\circledR}$ method and the broth breakpoint method (four treatments $=$ two culture procedures $\times$ two AST methodologies, $n=15$ birds/treatment). In trial 2, all Salmonella colonies were resistant to tetracycline (100\%) and most Salmonella colonies (>99\%) across treatments were susceptible to ciprofloxacin and sulfisoxazole. Results were not generated since insufficient variation in those data to perform ANOVA analysis.

\section{Discussion}

Poultry products and the poultry production environment are significant sources of Salmonella that may be resistant to antibiotics [16,17]. In the current study, Salmonella prevalence of the sampled broiler population was 28.6\% (59/206) in December 2018 and $50.0 \%$ (48/96) in August 2019. The Food Safety and Inspection Service (FSIS) tested 575 broiler ceca and reported a 17.9\% prevalence of Salmonella in 2014 [18]. Furthermore, FSIS tested whole carcasses and parts, Salmonella prevalence in whole carcasses, quarters, parts, and comminuted chicken was 3.59\%, 8.99\%, 8.36\%, 31.21\%, respectively, in 2019 [19]. Salmonella prevalence in carcass rinses has been reported as high as 54.09\% [19]. A survey of 15 broiler processing plants indicated that even after sanitization procedures, Salmonella prevalence remained at a range of $7.4 \%$ to $29.69 \%$ [20]. A research study indicated that $7 \%$ of poultry origin Salmonella isolates in the southeastern United States exhibited resistance to at least one antimicrobial [21]. The FSIS 2014 reported that 13\% of Salmonella isolates in 2014 were multidrug-resistant [18]. From 2015 to 2017, there was an increase in multidrugresistant Salmonella recovered from the chicken carcass $(9.5 \%$ to $18 \%)$ and chicken cecum samples (15\% to $25 \%)$ [22].

The NARMS established by the Centers for Disease Control and Prevention, USDA, and FDA, monitors Salmonella and other critical enteric bacteria in humans, animals, and meat products for their resistance to various antimicrobials of human and veterinary importance. However, according to the updated NARMS protocol [13], only one single Salmonella isolate per human source, retail meat, or food animal-related sample was assayed for AST via automated systems (Sensititre ${ }^{\mathrm{TM}}$ and Vitek 2 Compact). Limited isolates per biological sample are tested due to labor, consumables, and general expense, which brings up a concern about how mixed Salmonella populations containing clones that dominate during enrichment impact the surveillance program. A serotyping/sequencing method revealed $91 \%$ of poultry cecum samples harbored multiple serovars and one single sample could have four different serovars [23]. Thus, it is possible that examining a single isolate from one animal is not enough to determine the range of AMR patterns exhibited by Salmonella at premises. This study utilized an alternative method, a high throughput broth breakpoint assay, which allowed up to 94 isolates to be tested per plate with one antimicrobial compound at the CLSI/NARMS resistance breakpoint concentration [14]. By saving preparation time, antibiotics (other CLSI/NARMS recommended concentrations), and related consumables (plates, Mueller-Hinton broth, tips, etc.), the broth breakpoint assay is relatively inexpensive.

The broth breakpoint method reported significantly different AMR pattern determinations from a population of samples as compared to the paired outcomes on the Sensititre ${ }^{\circledR}$ platform in trial one. Increased resistance to chloramphenicol, ciprofloxacin, sulfisoxazole, and the combination of trimethoprim and sulfamethoxazole and decreased resistance to azithromycin, meropenem, and streptomycin were observed in poultry Salmonella when using the broth breakpoint method as compared to the Sensititre ${ }^{\circledR}$ method. A potential explanation for the difference observed in AMR patterns may be partially attributed to different isolate sample sizes. Increasing the number of isolates assayed could improve the 
overall detection of phenotypic AMR diversity in Salmonella within and between animal populations. However, exactly how many isolates should be included to improve coverage is unknown. This number will depend on the cost of the AST method used, the feasibility of sample collection, and the AMR diversity of the target bacteria.

When equalizing a sample size (Table 3 ) or limiting the sampling size to three isolates per RV enrichment sample (Table 4), the broth breakpoint assay still reported increased resistance to chloramphenicol, sulfisoxazole, and the combination of trimethoprim and sulfamethoxazole, and lower resistance to streptomycin as compared to the Sensititre ${ }^{\circledR}$ method. These observed differences in AMR patterns for the population may be attributable to diversity among the Salmonella isolates within and between individual ceca samples. Antimicrobial susceptibility testing methods have been largely developed to guide clinicians toward improved individual clinical treatment outcomes and are not generally conducive to high throughput testing for the purposes of surveillance and population research. The observations reported herein may therefore have important implications for understanding the underlying and emerging antimicrobial resistance trends in surveillance programs for agricultural animals.

Unexpected resistance to meropenem was found with both the Sensititre ${ }^{\circledR}$ method and breakpoint assay. Meropenem belongs to carbapenems, a critically important antibiotic class. In the current study, cecum samples were positive by PCR, however, not all Salmonella isolates were biochemically and serologically confirmed. A fault of the breakpoint method should be noted that it is not feasible to biochemically confirm every single isolate and that resistance could be inflated due to error processing morphologically similar isolates that were not Salmonella. While researchers were careful to pick individual and isolated colonies, it is also possible that mixed cultures may have caused some of these unanticipated results.

After attempting to account for the differences among Salmonella isolate diversity and sample size introduced to each assay, the broth breakpoint assay demonstrated similar susceptibility results to the Sensititre ${ }^{\circledR}$ method in trial two except for lower resistance to gentamicin $(0.00 \%$ vs. $3.75 \%)$ and chloramphenicol $(0.00 \%$ vs. $2.08 \%)$ and a higher resistance to nalidixic acid (5.0\% vs. $0.84 \%)$, respectively. These differences may have been caused by methodological variations of the two AST methods, including antimicrobial compound preparation (dry powder in the Sensititre ${ }^{\circledR}$ plate vs. wet solution in the broth breakpoint plate), inoculum preparation (overnight incubation onto blood agar plates vs. in the 96-well plate with $\mathrm{MH}$ broth), reaction volume $\left(50 \mu \mathrm{L}\right.$ in each well of the Sensititre ${ }^{\circledR}$ plate vs. $200 \mu \mathrm{L}$ in each well of broth breakpoint ), reaction concentration $\left(1.4 \times 10^{5} \mathrm{cfu} / \mathrm{mL}\right.$, estimated by McFarland standards vs. $1.5 \times 10^{6} \mathrm{cfu} / \mathrm{mL}$, estimated by $\mathrm{OD}_{600}$ reading), and interpretation of AMR results (Vizion ${ }^{\mathrm{TM}}$ Digital MIC Viewing System vs. OD600 reading). In early comparative studies, the Sensititre ${ }^{\circledR}$ platform was compared to other susceptibility tests (MicroScan, Vitek2, E-test, and Micronaut strip) for their essential and categorical agreements with broth micro-dilution method [24-27]. The Sensititre ${ }^{\circledR}$ method exhibited $97.1 \%$ essential agreement with the reference broth micro-dilution method in measuring Klebsiella pneumoniae resistance to polymyxins and 92.5\% for colistin [26], and acceptable categorical agreements $(>90 \%)$ in measuring colistin and polymyxin B resistance in Enterobacteriaceae [24,27]. No major errors were found with the Sensititre ${ }^{\circledR}$ system [25]. Other antimicrobial susceptibility methodologies like the E-test and disk-diffusion were also considered less accurate than the reference agar dilution procedure [28,29]. Further research is warranted to determine the comparative accuracy of the high throughput broth breakpoint assay as compared to agar/broth dilution methods [30] when considering high volumes of diverse isolate testing.

Another important finding in this study was that observed resistance to multiple antimicrobial compounds was associated with culture methods. Salmonella detection and isolation bias imparted by microbiological culture methods and media have been documented and potential implications to the accuracy of surveillance programs were discussed [31-34]. Singer et al. [31] reported cultivation media selected preferentially for specific strains of Salmonella in heterogeneous cultures, such as a tetrathionate-RV 
protocol and a bovine fecal culture. A Salmonella Newport strain was more competitive than two Salmonella Typhimurium and one Salmonella Enteritidis strains in both media [31]. Concentrations of Salmonella Enteritidis at the end of the enrichment period were eight-fold higher in tetrathionate as compared to those in RV, whereas Salmonella Schwarzengrund and Reading preferentially enriched in RV [32]. Goriski [33] reported the bias of selective enrichment media (RV and RV nutrient-dense version) on the types of Salmonella isolates from mixed strain culture and cattle fecal cultures. Different formulations of RV media reported different patterns of strain dominance [33]. Metagenomic analyses indicated that the enrichment procedure reported significantly different taxonomic profiles with high numbers of putative Salmonella sequences as compared to the unenriched samples [34]. A serotype sequence assay revealed the RV enrichment reduced the Salmonella serovar diversity [32]. However, whether the RV enrichment altered Salmonella AMR prevalence by selectively favoring the growth of certain subgroups of Salmonella or directly selecting AMR determinants is unclear.

\section{Conclusions}

Our study measured AMR prevalence in broiler cecum collected from a slaughter plant. These data suggest that the breakpoint method allowed more Salmonella isolates to be tested resulting in significantly different AMR patterns than the automated Sensititre ${ }^{\circledR}$ platform. We found that the ability to test more isolates was more representative of the diverse microbial population. This research also demonstrates that the selectivity imparted by pre-enrichment and selective secondary enrichment in RV broth may indeed bias the Salmonella detected and isolated, and therefore, subsequently bias the AMR patterns determined through susceptibility testing. While enrichment procedures are necessary to detect Salmonella and estimate true underlying prevalence in a population, our data suggest that surveillance and determination of AMR patterns for a population could be severely limited due to the bias imparted by the isolation procedure and AST methodology combined.

Author Contributions: Conceptualization, W.E.C., H.O.P. and M.B.F.; Data curation, X.W.; Formal analysis, X.W., W.E.C., H.O.P., T.J.J. and J.P.M.; Funding acquisition, W.E.C. and H.O.P.; Investigation, X.W., T.J.J., J.A.B., J.P.M., Y.Z.F., A.P.M. and M.B.F.; Methodology, W.E.C., H.O.P., T.J.J. and J.A.B.; Project administration, X.W. and M.B.F.; Resources, J.P.M.; Supervision, M.B.F.; Writing-original draft, X.W. and M.B.F.; Writing-review \& editing, W.E.C., H.O.P., T.J.J., J.A.B., J.P.M. All authors have read and agreed to the published version of the manuscript.

Funding: This research was graciously funded by Diamond V; Texas A\&M University proposal \# 06-408414-91300.

Institutional Review Board Statement: All experimental procedures were reviewed and approved by the Texas A\&M University Institutional Biosafety Committee (IBC number: 2019-073). All samples were collected post-mortem from a commercial poultry processing plant, therefore an animal use protocol was not required for this study.

Acknowledgments: We thank Kathleen Andrews and Denise Caldwell for technical assistance with the Sensititre ${ }^{\circledR}$ assay.

Conflicts of Interest: The authors declare no conflict of interest.

\section{References}

1. Antunes, P.; Mourao, J.; Campos, J.; Peixe, L. Salmonellosis: The role of poultry meat. Clin. Microbiol. Infect. 2015, 22, 110-121. [CrossRef]

2. Cosby, D.E.; Cox, N.A.; Harrison, M.A.; Wilson, J.L.; Buhr, R.J.; Fedorka-Cray, P.J. Salmonella and antimicrobial resistance in broilers: A review. J. Appl. Poult. Res. 2015, 24, 408-426. [CrossRef]

3. Pan, Z.; Wang, X.; Zhang, X.; Geng, S.; Chen, X.; Pan, X.W.; Cong, Q.; Liu, X.; Jiao, X.; Liu, X. Changes in antimicrobial resistance among Salmonella enterica subspecies enterica serovar Pullorum isolates in China from 1962 to 2007. Vet. Microbiol. 2009, 136, 387-392. [CrossRef] [PubMed]

4. Nhung, N.T.; Chansiripornchai, N.; Carrique-Mas, J.J. Antimicrobial resistance in bacterial poultry pathogens: A review. Front. Vet. Sci. 2017. [CrossRef] [PubMed] 
5. Food and Drug Administration. FDA Releases Five-Year Plan for Supporting Antimicrobial Stewardship in Veterinary Settings. Available online: https:/ /www.fda.gov/animal-veterinary/cvm-updates/fda-releases-five-year-plan-supporting-antimicrobialstewardship-veterinary-settings (accessed on 28 October 2020).

6. Jajere, S.M. A review of Salmonella enterica with particular focus on the pathogenicity and virulence factors, host specificity and antimicrobial resistance including multidrug resistance. Vet. World 2019, 12, 504-521. [CrossRef]

7. Chuanchuen, R.; Padungtod, P. Antimicrobial resistance genes in Salmonella enterica isolates from poultry and swine in Thailand. J. Vet. Med. Sci. 2009, 71, 1349-1355. [CrossRef]

8. United State Department of Agriculture. National Antimicrobial Resistance Monitoring System (NARMS). 2019. Available online: https: / /www.fsis.usda.gov/wps/portal/fsis/topics/data-collection-and-reports/microbiology/antimicrobial-resistance/ narms (accessed on 20 October 2020).

9. Food and Drug Administration. NARMS Genomic Data; Department of Health and Human Services: Rockville, MD, USA, 2017; Available online: https:/ / www.fda.gov/animal-veterinary/national-antimicrobial-resistance-monitoring-system/narmsgenomic-data (accessed on 31 October 2020).

10. Pornsukarom, S.; van Vliet, A.H.M.; Thakur, S. Whole genome sequencing analysis of multiple Salmonella serovars provides insights into phylogenetic relatedness, antimicrobial resistance, and virulence markers across humans, food animals and agriculture environmental sources. BMC Genom. 2018, 19, 801. [CrossRef] [PubMed]

11. Louie, M.; Cockerill, F.R. Susceptibility testing. Phenotypic and genotypic tests for bacteria and mycobacteria. Infect. Dis. Clin. North Am. 2002, 15, 1205-1226. [CrossRef]

12. Reller, L.B.; Weinstein, M.; Jorgensen, J.H.; Ferraro, M.J. Antimicrobial susceptibility testing: A review of general principles and contemporary practices, clinical infectious diseases. Clin. Infect. Dis. 2009, 49, 1749-1755.

13. National Antimicrobial Resistance Monitoring System (NARMS). Manual of Laboratory Methods, 3rd ed; 2016. Available online: https: / / www.fda.gov/media/101423/download. (accessed on 20 February 2021).

14. Feye, K.M.; Anderson, K.L.; Scott, M.F.; Mcintyre, D.R.; Carlson, S.A. Inhibition of the virulence, antibiotic resistance, and fecal shedding of multiple antibiotic resistant Salmonella Typhimurium in broilers fed original XPC ${ }^{\mathrm{TM}}$. Poult. Sci. 2016, 95, 2902-2910. [CrossRef]

15. SAS Institute. SAS Proprietary Software Release 9.2; SAS Inst. Inc.: Cary, NC, USA, 2010.

16. Andoh, L.A.; Dalsgaard, A.; Obiri-danso, K.; Newman, M.J.; Barco, L.; Olsen, J.E. Prevalence and antimicrobial resistance of Salmonella serovars isolated from poultry in Ghana. Epidemiol. Infect. 2016, 144, 3288-3299. [CrossRef] [PubMed]

17. Abdi, R.D.; Mengstie, F.; Beyi, A.F.; Beyene, T.; Waktole, H.; Mammo, B.; Ayana, D.; Abunna, F. Determination of the sources and antimicrobial resistance patterns of Salmonella isolated from the poultry industry in Southern Ethiopia. BMC Infect. Dis. 2017, 17, 352. [CrossRef] [PubMed]

18. Food Safety and Inspection Service. National Antimicrobial Resistance Monitoring System Cecal Sampling Program. 2014. Salmonella Report. Available online: https:/ /www.fsis.usda.gov/node/1974 (accessed on 25 March 2021).

19. Food Safety and Inspection Service. FSIS Data: Sampling Project Results. 2021. Available online: https://www.fsis.usda.gov/ sites/default/files/media_file/2021-03/sampling_project_results_data.pdf (accessed on 25 March 2021).

20. Obe, T.; Nannapaneni, R.; Shilling, W.; Zhang, L.; McDaniel, C.; Kiess, A. Prevalence of Salmonella enterica on poultry processing equipment after completion of sanitization procedures. Poult. Sci. 2020, 99, 4539-4548. [CrossRef] [PubMed]

21. Velasquez, C.G.; Macklin, K.S.; Kumar, S.; Bailey, M.; Ebner, P.E.; Olive, H.S.; Martin-Gonzalez, F.S.; Singh, M. Prevalence and antimicrobial resistance patterns of Salmonella isolated from poultry farms in southeastern United States. Poult. Sci. 2018, 97, 2144-2152. [CrossRef]

22. Food and Drug Administration. 2016-2017 NARMS Integrated Summary. 2017. Available online: https:/ / www.fda.gov / animalveterinary/national-antimicrobial-resistance-monitoring-system/2016-2017-narms-integrated-summary (accessed on 25 March 2021).

23. Cameron, P.T.; Doak, A.N.; Amirani, N.; Schroeder, E.A.; Wright, J.; Kariyawasam, S.; Lamendella, S.; Shariat, N.W. Highresolution identification of multiple Salmonella serovars in a single sample by using CRISPR-SeroSeq. Food Microbiol. 2018. [CrossRef]

24. Chew, K.L.; La, M.; Lin, R.T.P.; Teo, J.W.P. Colistin and polymyxin B susceptibility testing for carbapenem-resistant and mcrpositive Enterobacteriaceae: Comparison of Sensititre, MicroScan, Vitek2, and E-test with broth microdilution. J. Clin. Microbiol. 2017, 55, 2609-2616. [CrossRef]

25. Jayol, A.; Nordmann, P.; Andre, C.; Poirel, L.; Dubois, V. Evaluation of three broth microdilution systems to determine colistin susceptibility of Gram-negative bacilli. J. Antimicrob. Chemother. 2018, 73, 1272-1278. [CrossRef]

26. Richter, S.S.; Karichu, J.; Otiso, J.; Heule, H.; Keller, G.; Cober, E.; Rojas, L.J.; Hujer, A.M.; Hujer, K.M.; Marshall, S.; et al. Evaluation of Sensititre broth microdilution plate for determining the susceptibility of carbapenem-resistance Klesiella pneumoniae to polymyxins. Diagn. Microbiol. Infect. Dis. 2018, 91, 89-92. [CrossRef]

27. Yusuf, E.; Westreenen, M.; Goessens, W.; Croughs, P. The accuracy of four commercial broth microdilution tests in the determination of the minimum inhibitory concentration of colistin. Ann. Clin. Microbiol. Antimicrob. 2020, 19, 42. [CrossRef]

28. Ogata, S.K.; Gales, A.C.; Kawakami, E. Antimicrobial susceptibility testing for Helicobacter pylori isolates from Brazilian children and adolescents: Comparing agar dilution, E-test, and disk diffusion. Braz. J. Microbiol. 2014, 45, 1439-1448. [CrossRef] [PubMed] 
29. Barberis, C.M.; Sandoval, E.; Rodriguez, C.H.; Ramires, M.S.; Famiglietti, A.; Almuzara, M.; Vay, C. Comparison between disk diffusion and agar dilution methods to determine in vitro susceptibility of Coryebacterium spp. clinical isolates and update of their susceptibility. J. Glob. Antimicrob. Resist. 2018, 14, 246-252. [CrossRef] [PubMed]

30. CLSI. Performance Standards for Antimicrobial Susceptibility Testing, 27th ed.; CLSI Supplement M100; Clinical and Laboratory Standards Institute: Wayne, PA, USA, 2017.

31. Singer, R.S.; Mayer, A.E.; Hanson, T.E.; Issacson, R.E. Do microbial interactions and cultivation media decrease the accuracy of Salmonella surveillance systems and outbreak investigations? J. Food Prot. 2009, 72, 707-713. [CrossRef] [PubMed]

32. Cox, N.A.; Berrang, M.E.; House, S.L.; Medina, D.; Cook, K.L.; Shariat, N.W. Population analyses reveal pre-enrichment method and selective enrichment media affect Salmonella serovars detected on broiler carcasses. J. Food Prot. 2019, 8, 1688-1696. [CrossRef] [PubMed]

33. Goriski, L. Selective enrichment media bias the types of Salmonella enterica strains isolated from mixed strain cultures and complex enrichment broths. PLoS ONE 2012, 7, 4. [CrossRef] [PubMed]

34. Pettengill, J.B.; McAvoy, E.; White, J.R.; Allard, M.; Brown, E.; Ottesen, A. Using metagenomic analyses to estimate the consequences of enrichment bias for pathogen detection. BMC Res. Notes 2012, 5, 378. [CrossRef] 